\title{
Heavy quark \& direct photon production and heavy quark parton densities
}

\author{
T. Stavreva
}

Laboratoire de Physique Subatomique et de Cosmologie, UJF, CNRS/IN2P3, INPG, 53 avenue des Martyrs, 38026 Grenoble, France

\begin{abstract}
Direct photon production in association with a heavy quark can serve as an excellent tool for the study of the heavy quark distributions. Currently it is believed that heavy quarks are produced radiatively inside the nucleus, and so there is no need to take into account heavy quark parameters inside global PDF analysis. Certain models taking into account the possibility of an intrinsic charm component exist. Here we present how these affect the $\gamma+c$ cross section. While at $p A$ collisions the potential of this process to constrain the gluon nuclear PDF which is currently largely unconstrained is presented.
\end{abstract}

Keywords: direct photon, heavy quark, PDF

\section{Introduction}

\subsection{Direct $\gamma+Q$}

In order to gather more information on the heavy quark PDFs it is important to investigate in detail processes sensitive to them. Direct photon production in association with a heavy quark (c or b) is exactly one such process due to its dependence on subprocesses sensitive to the initial state heavy quark (HQ) distributions [1].

At LO it has a simple form - it consists of one hardscattering subprocess, $g+Q \rightarrow \gamma+Q$ (Compton subprocess), and also fragmentation contributions these being greatly suppressed due to experimental isolation requirements. At NLO the number of contributing hardscattering subprocesses increases to seven 2 ,

$$
\begin{array}{ll}
g+g \rightarrow Q+\bar{Q}+\gamma & Q+Q \rightarrow Q+Q+\gamma \\
g+Q \rightarrow g+Q+\gamma & Q+\bar{Q} \rightarrow Q+\bar{Q}+\gamma \\
Q+q \rightarrow q+Q+\gamma & q+\bar{q} \rightarrow Q+\bar{Q}+\gamma \\
Q+\bar{q} \rightarrow Q+\bar{q}+\gamma &
\end{array}
$$

also including NLO fragmentation effects, which are small due to the isolation cuts as in the LO case. Given that most of the subprocesses are initiated by either

\footnotetext{
Email address: stavreva@lpsc.in2p3.fr (T. Stavreva)

${ }^{1}$ The LO fragmentation contributions consist of all $2 \rightarrow 2$ subprocesses of order $O\left(\alpha_{s}^{2}\right)$ containing at least one heavy quark in the final state convoluted with the photon fragmentation function $D_{\gamma / q, g}\left(z, Q^{2}\right)$.

${ }^{2}$ This calculation is performed in the variable flavor number scheme, where the heavy quarks are treated as massless.
}

heavy quarks or gluons, and in the proton the gluon distribution is well known, it is possible to focus on constraining the heavy quark PDF through measurements of this process.

\subsection{Intrinsic Charm}

In the standard global analysis of PDFs, heavy quarks do not have free fit parameters associated with them. Therefore currently it is assumed that there is no intrinsic charm (IC) or intrinsic bottom (IB) inside the nucleus. This means that the heavy quark PDF can be obtained purely perturbatively through the use of the DGLAP evolution equations, having set an appropriate initial condition ( such as $f_{Q}\left(x, Q_{0}=m_{Q}\right)=0$ ). However there is no theoretical reason that this should be the case and moreover there have been some data that point towards the existence of an intrinsic charm component in the proton $\left(F_{2}^{c}\right.$ at large $x$ as measured by EMC [2]). As a result there are non-perturbative models predicting the size and shape of the intrinsic heavy quark component [3, 4] which are used in PDF global fits [5] 3 In Fig. 1 the difference between different IC PDFs and the radiatively radiate charm (CTEQ6.6M) is displayed. Both the BHPS (dashed red line) and Meson Cloud model (dotted green line) display a similar behavior where they peak at high $x$, while being very similar to the radiatively generated charm (solid black line)

\footnotetext{
${ }^{3}$ Currently there are fits available only for IC. The IB/IC ratio is expected to be proportional to $m_{c}^{2} / m_{b}^{2}$.
} 
at lower $x$. The purely phenomenologically inspired sea-like model (dash-dotted blue line) produces a PDF which is larger than the radiatively generated one in a broad $x$ range. In order to constrain precisely the HQ $\mathrm{PDF}$ it is important to measure processes sensitive to it such as $\gamma+Q$. How these different models affect the $\gamma+Q$ cross-section is presented in the next section.

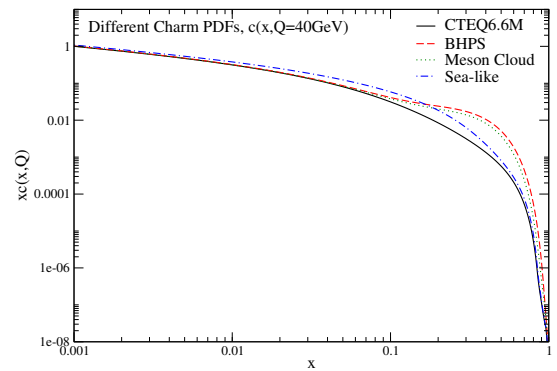

Figure 1: Comparison between the radiatively generated charm PDF (CTEQ6.6M, solid black line), charm PDF including IC: under the BHPS model (CTEQ6.6C1, dashed red line), under the Meson Cloud model (CTEQ6.5C4, dotted green line), under the sea-like model (CTEQ6.6C3, dash-dotted blue line)

\section{Predictions: hadron-hadron collisions}

\subsection{Tevatron}

The differential cross-section vs $p_{T \gamma}$ for $\gamma+c$ and $\gamma+b$ production at the Tevatron is presented at both NLO and LO in Fig. 2 From there we see that the difference between $d \sigma^{\gamma+c} / d p_{T \gamma}$ and $d \sigma^{\gamma+b} / d p_{T \gamma}$ at LO stays almost constant, whereas it decreases at NLO with growing $p_{T \gamma}$. The reason for this is that at the Tevatron, due to the abundance of valance quarks and anti-quarks, the annihilation subprocess $(q+\bar{q} \rightarrow Q+\bar{Q}+\gamma)$ starts to dominate the cross-section, and this subprocess is exactly the same for both $\gamma+c$ and $\gamma+b$ production. When one looks at the comparison between theory and experimental measurements (performed by the D $\varnothing$ collaboration [6] ) in Fig. 3, the agreement for $\gamma+b$ is very good, whereas for $\gamma+c$ the data overshoot the theoretical predictions at high $p_{T}$. One possibility to try and correct for this discrepancy is to utilize IC PDFs in the theoretical predictions. In Fig. 4 the data over theory ratio is presented, as well as the ratios $\frac{d \sigma^{B H P S} / d p_{T \gamma}}{d \sigma^{C T E Q 6.6 M} / d p_{T \gamma}}$, $\frac{d \sigma^{\text {sea-like }} / d p_{T \gamma}}{d \sigma^{C T E Q 6.6 M} / d p_{T \gamma}}$. As expected the sea-like cross-section overshoots the data at low $p_{T}$, but undershoots it at high $p_{T}$. Conversely the BHPS cross-section, follows the data trend, and provides a better description than both the radiatively generated charm and the sea-like charm cross-section, however it still undershoots the data at very high $p_{T}$. Therefore in order to confirm or disprove the existence of IC more data for processes sensitive to IC is needed. In the next subsection the effects of IC on the direct photon and heavy quark cross-section, and the possibility of constraining IC at the LHC are presented.

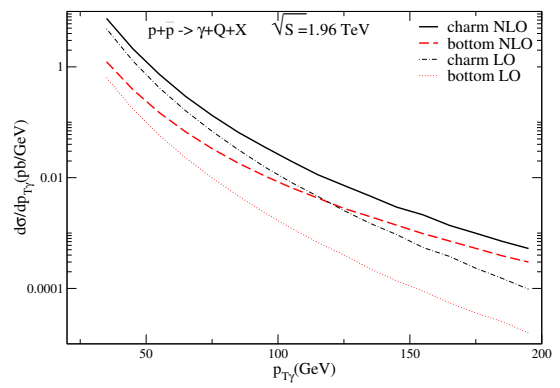

Figure 2: $d \sigma^{\gamma+Q} / d p_{T \gamma}$ : for charm at NLO (black solid line), at LO (black dash-dotted line), for bottom at NLO (red dashed line), at LO (red dotted line).

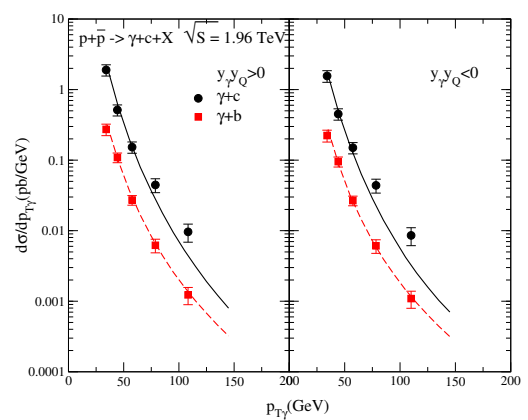

Figure 3: Comparison between theory $-\gamma+c$ (solid black curve), $\gamma+b$ (dashed red curve) and $\mathrm{D} \varnothing$ data $-\gamma+c$ (black circles), $\gamma+b$ (red squares)

\section{2. $\mathrm{LHC}$}

At the LHC, already at $7 \mathrm{TeV}$ the $x$ probed is small $\left(x \sim p_{T} / \sqrt{S}\right)$. Therefore in order to be sensitive to the BHPS IC, which shows up at large- $x$ (Fig. 1), one has to probe forward rapidities in order to be sensitive to it. Utilizing experimental cuts appropriate for the CMS detector [7, 8] and focusing on the forward rapidity region $\left(1.566<\left|y_{\gamma}\right|<2.5,1.5 \leq\left|y_{Q}\right|<2.0\right)$ for both photons and c-quarks, we present in Fig. 5 the predictions for $d \sigma^{\gamma+c} / d p_{T \gamma}$ at $\sqrt{S}=7 \mathrm{TeV}$. The BHPS crosssection (green dashed line) is similar to the radiatively 


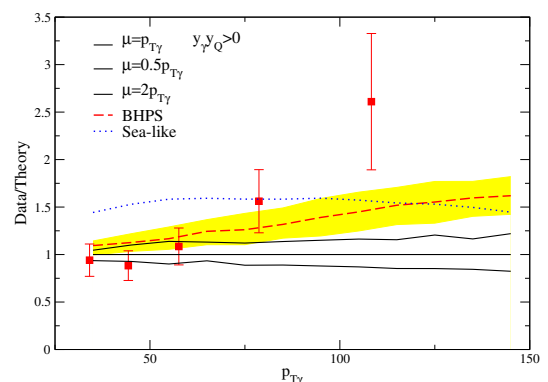

Figure 4: Data over theory ratio for $\gamma+c$ production (red squares), the theoretical predictions are for radiatively generated charm including the scale dependence (black lines), BHPS IC (red dashed line), sealike IC (blue dotted line).

generated one (black solid curve) at low $p_{T}$, but as $p_{T}$ increases (and correspondingly $x$ increases) so does the difference between the two curves, thus providing a possibility for testing the existence of BHPS IC. To portray the need for measurements at forward rapidities the ratio between the BHPS cross-section and the radiatively generated one for several different combinations of the photon and heavy quark rapidities is presented in Fig. 6. There the ratio at larger $p_{T}$ increases from $\sim 1.1$ for $\left|y_{\gamma}\right|<1.4442 \&\left|y_{Q}\right|<0.5$, to up to $\sim 2.2$ for $1.566<\left|y_{\gamma}\right|<2.5 \& 1.5 \leq\left|y_{Q}\right|<2.0$.

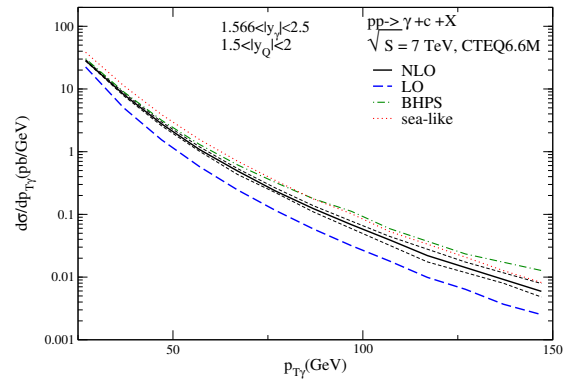

Figure 5: $d \sigma^{\gamma+c} / d p_{T \gamma}$ at CMS for forward rapidity for radiatively generated charm (black solid line) + scale dependence (black dashed curves), BHPS IC (green dash-dotted line), sea-like IC (red dotted line).

\section{Predictions: hadron-ion collisions}

It is also interesting to study the IC effects in $p A$ collisions. However in order for such a study to be performed, the gluon nuclear PDF (nPDF) should be as well known as in the free proton. Unfortunately

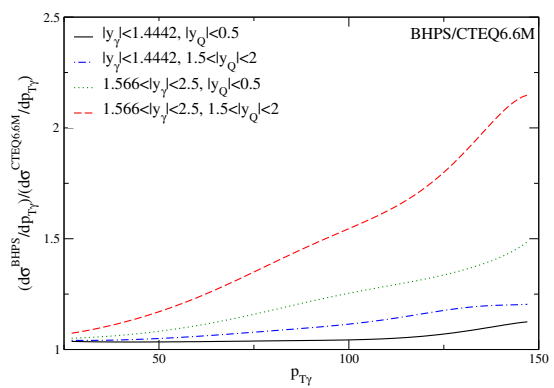

Figure 6: Ratio of BHPS IC $\gamma+c$ cross-section over one using radiatively generated charm for different restrictions on the photon and charm rapidity.

this is not the case, and the gluon distribution is only very weakly constrained by NMC data on $F_{2}^{D}\left(x, Q^{2}\right)$ and $F_{2}^{S n} / F_{2}^{C}\left(x, Q^{2}\right){ }^{4}$ The large uncertainty in the nuclear gluon as well as the variations in predictions of different fits (nCTEQ [9, 10, 11], HKN07 (red dashdotted band) [12], EPS09 [13]) is presented in Fig. 7 in the form of the gluon nuclear modification factor $R_{g}(x, Q)=g^{p / P b}(x, Q) / g^{p}(x, Q)$. It is quite clear that there is a strong need for data constraining the gluon nPDF. In the general framework, where there is no IC considered, and the charm distribution is solely based on the gluon PDF, the charm nuclear modification, $R_{c}$, follows $R_{g}$ quite closely as shown in Fig. 8 Since $\sigma^{\gamma+c}$ is quite sensitive to the gluon PDF as well as the charm PDF as shown in Fig. 9, where the dominating subprocesses at $\sqrt{S}=8.8 \mathrm{TeV}$ for $p-A$ collisions are $g g$ and $g Q$ initiated, it is a very useful process for constraining the gluon nPDF. Focusing on the central rapidity region in ALICE will isolate any effects caused by the possible presence of the theoretically favored BHPSIC, which will appear at large- $x$ and forward rapidity, Fig. 6. Therefore we can expect that the nuclear corrections to $\sigma^{\gamma+c}$ will follow the ones for $R_{g}$ and $R_{c}$ correspondingly. In Fig. 10 the nuclear modification factor, $R^{\sigma^{\gamma+c}}=\frac{d \sigma / d p_{T \gamma}(p A)}{A_{P b} d \sigma / d p_{T \gamma}(p p)}$, to the direct photon and heavy quark cross-section is shown. It can be seen clearly by comparing Fig. 10 and the boxed region in Fig. 7 that the nuclear modification factor to the cross-section, $R^{\sigma^{\gamma+c}}$, follows closely the nuclear modifications for the gluon $\mathrm{nPDF}\left(R_{g}\right)$, in the region of $\mathrm{x}$ probed at the LHC; for more details see [15]. Therefore this process is an excellent candidate for constraining the gluon nuclear distribution.

\footnotetext{
${ }^{4}$ This is true for most nPDF fits, while EPS09 also includes data on $\pi^{0}$ production at RHIC.
} 


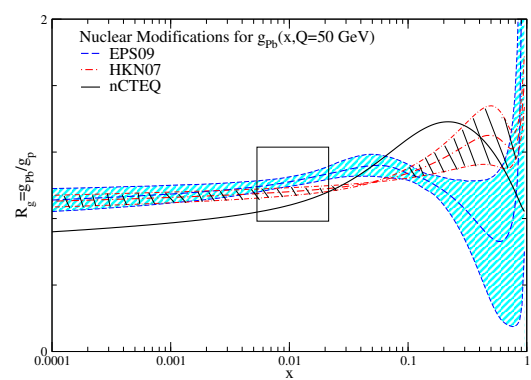

Figure 7: The gluon nuclear modification factor for nCTEQ (solid black line), HKN07 (red dash-dotted band), EPS09 (dashed blue band).

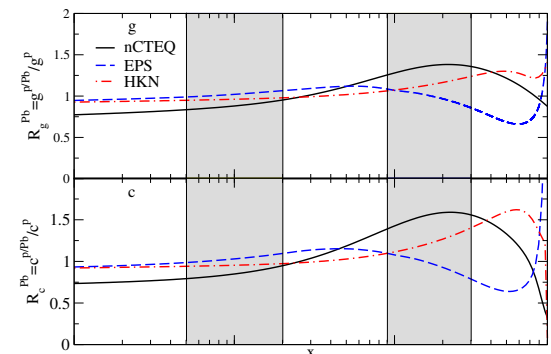

Figure 8: Comparison between the charm and gluon nuclear modification factors for nCTEQ (solid black line), HKN07 (red dash-dotted band), EPS09 (dashed blue band), the two shaded regions represent the $x$ probed at LHC (first band) and at RHIC (second band).

\section{Conclusion}

It was shown that through direct photon production in association with a heavy quark we can study and constrain the heavy quark PDFs. More specifically measurements at the Tevatron of this process have indicated a possible existence of IC that seems to favor the BHPS model. Further tests at the LHC of this process especially at forward rapidities should be able to constrain or disfavor the presence of intrinsic charm in the proton. While such a constrain inside the nucleus is also very important, one has to have a quite precisely determined gluon nPDF in order to do so. Since this is not the case, we have shown here that direct photon + heavy quark production at $\mathrm{pA}$ collisions is also a great process for constraining the nuclear gluon PDF.

\section{References}

[1] T. Stavreva, J.F. Owens, Phys. Rev. D79, 054017 (2009), [0901.3791 [hep-ph]].

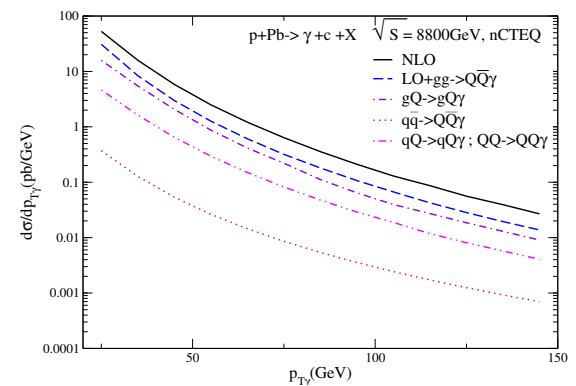

Figure 9: Subprocess contributions to $\sigma^{\gamma+c}$ at p-A collisions at ALICE.

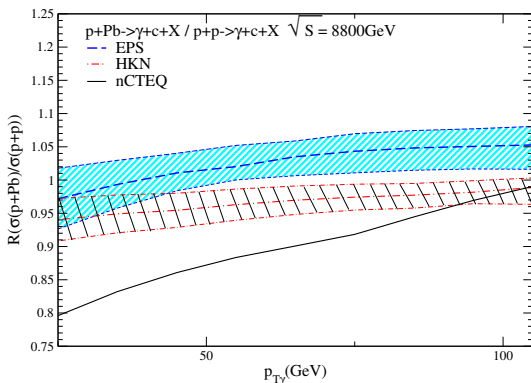

Figure 10: Nuclear modification factor for $\gamma+c$ at ALICE, using nCTEQ (black solid line), EPS09 (blue dashed line) + error band, HKN07 (red dash-dotted line) + error band

[2] J. J Aubert et al., [European Muon Collaboration], Nucl. Phys. B213, 31 (1983).

[3] S. J. Brodsky et al., Phys. Lett. B93, 451 (1980).

[4] F. S. Navarra et al., Phys. Rev. D54, 842 (1996).

[5] J. Pumplin et al., Phys. Rev. D75, 054029 (2007) |hepph/0701220.

[6] V. M. Abazov et al., [DØ Collaboration], Phys. Rev. Lett. 102, 192002 (2009) [0901.0739 [hep-ex]].

[7] [CMS Collaboration] CMS note: CMS PAS EGM-10-005, available on the CERN CDS information server.

[8] [CMS Collaboration] CMS note: CMS PAS BPH-10-009, available on the CERN CDS information server.

[9] I. Schienbein et al., Phys. Rev. D77 054013 (2008), [0812.3370 [hep-ph]].

[10] I. Schienbein et al., Phys. Rev. D80 094004 (2009), [0907.2357 [hep-ph]].

[11] K. Kovarik et al., [1012.0286 [hep-ph]]

[12] M. Hirai et al., Phys. Rev. C76 065207 (2007) [0709.3038 [hep$\mathrm{ph}]]$.

[13] K. J. Eskola et al., JHEP 07102 (2008).

[14] A. Abulencia et al., [CDF Collaboration], Phys. Rev. Lett. 99, 132001 (2007)

[15] T. Stavreva et al., [1012.1178 [hep-ph]] 